\title{
Root Topographic Study on Molars with Furcation Involvements in Taiwanese Adults: A Risk Analysis of Furcation Entrance Dimension and Root Trunk Length
}

\author{
Guey-Lin $\mathrm{Hou}^{1,2 *}$ \\ ${ }^{1}$ Former Professor, Graduate Institute of Dental Sciences and Department of Periodontics, School of Dental Medicine, Kaohsiung Medical \\ University, Kaohsiung City, Republic of China \\ ${ }^{2}$ Former Chairman and Professor, Dental Department, Periodontal Prosthetic Center, Chang-Gung Memorial Hospital, Kaohsiung City, Taiwan, \\ Republic of China
}

*Corresponding author: Guey-Lin Hou, Former Professor, Graduate Institute of Dental Sciences and Department of Periodontics, School of Dental Medicine, Kaohsiung Medical University, Kaohsiung City, Republic of China, Tel: 0088677472606; E-mail: hougl2001@yahoo.com.tw

Received: 17 Aug, 2020 | Accepted: 27 Aug, 2020 | Published: 02 Sep, 2020

Citation: Hou GL (2020) Root Topographic Study on Molars with Furcation Involvements in Taiwanese Adults: A Risk Analysis of Furcation Entrance Dimension and Root Trunk Length. Int J Dent Oral Health 6(6): dx.doi.org/10.16966/2378-7090.337

Copyright: @ 2020 Hou GL. This is an open-access article distributed under the terms of the Creative Commons Attribution License, which permits unrestricted use, distribution, and reproduction in any medium, provided the original author and source are credited.

\begin{abstract}
The present study was to investigate the Topography of Root Trunk type (RTT) and Furcation Entrance Dimension (FED) on extracted molars affected with advanced periodontitis and furcation involvement (FI). A total 169 extracted maxillary and mandibular molars (165 patients) affected with severely advanced periodontal destruction. The RTT was categorized as A, B, C, and the FED was clarified into subclass $-1,-2$, and -3 , respectively. Results showed that the prevalence of mandibular second molars with RTT-C and FED-1 occurs higher; (33.0\%), and the relationship between RTT and FED is significance $(p<0.005)$. In addition, the relationship between RTT-C and FED-1 also shows a statistical significance $(p<0.01)$ on the buccal surface $(41.5 \%)$ of the mandibular second molar.

It was concluded that teeth with RTT-C and FED-1, affected the highest risk at the mandibular second molars with Class III FI as compared to other molars during evaluation of periodontal prognosis.
\end{abstract}

Keywords: FED; RTT; Class III molar Fl; Risk factor; Root anatomy

\section{Introduction}

It is now well-known that some anatomic factors on molar furcation, such as root concavities, Cervical Enamel Projections (CEP), Molar Root Fusion (RF), Intermediate Bifurcational Ridges (IBR), and Shallow Furcation Entrance Dimension (FED) $(<0.56 \mathrm{~mm}$ or $<0.75 \mathrm{~mm}$ ) have a greater risk for the treatment of furcation defects as compared to teeth without furcation involvement [1-4].

Understanding of external molar root morphology is of most importance in periodontal therapy, not only in the evaluation of the prognosis, but also in the selection of treatment modalities of furcation involvement [5]. Addressed that the significance regarding macroscopic and microscopic topographic structures of the long root trunk and narrow furcation entrance at the molar area might also play a pivotal role in the disease progression. In addition, they also, in a retrospective study, emphasized that tooth type and degree of FI had been considered by the majority of practitioners as the most important factors influencing the decision making of treatment choice. Recently, long-term retrospective studies indicated, that the significance of periodontal regenerative procedures in the treatment of advanced furcation involvements was rather limited in the maxillary molar areas [6-8]. Fleischer HC, et al. [8] reported that there existed only a calculus-free surface in only $68 \%$ of cases even flap operation was performed by an experienced operator when furcation aspects were assessed. Similar observations were also documented by several investigators [9-10]. This poor outcome might be explained by the anatomic complexities of various depths of root grooves, accessory canals, intermediate bifurcational ridges and enamel projections within the furcal areas. The fact reflecting that instrumentation in this area of the root is difficult $[2,11]$.

Previous studies have reported the intimate relationship between anatomical topography relative to the outcome of periodontal therapy in molar furcation involvement $[2,12-15]$. Pontoriero $\mathrm{R}$ and Lindhe $\mathrm{J}$ [15] Based on the conclusion of their reports, the tortuous regions, and the proximity to the furcation entrance which were inaccessible to mechanical cleaning, seemed to be a high risk factor for disease 
progression in furcation areas. Recently, a series of studies with respect to the detailed topography of furcation entrance architecture and variations in molar root trunks in correlation with the molar location, type and dimensions, were also documented [2,3,16-19]. Based on our data of the first and second molars affected with the Class III of furcation involvement, there was a significant difference ( 3 folds) in Periodontal Attachment Loss (PAL) between RTT type A and type $\mathrm{C}$ [3]. Our further study on the types of root trunk and dimensions with respect to the periodontal attachment level revealed that this data might be considered as a diagnosing aid in decision making for Class III FIs [18]. In addition, combination of the types of root trunks and the degree of molar FI might be useful in the clinical evaluation of molar diagnosis, prognosis and treatment plan $[18,19]$. All of the these reports suggested that a decision making for molar root morphology, including the combination of furcation entrance dimension and type of root trunk in correlation with the FI of the periodontally involved molars may be more important in the prognosis, diagnosis and treatment plan $[2,13,17,19-21]$.

In some reports, molars with long root trunks did not easily develop FI as compared to those with short root trunks, the periodontal complications, such as severe PAL with a resultant molar loss, might still occur when such molars having RTT with type B or C $[2,3,18,19]$. However, what role, if any, the simultaneous occurrence of morphologic variations of Furcation Entrance Dimension (FED) and root trunk length plays in the progression and prognosis of periodontal disease within molar furcation has not been adequately assessed due to little or no clinical study of adding FED together with RTT. The purpose of this study was to investigate accumulative effect of variation of FED and RTT on extracted molars with severe Class III FI. In addition, whether the association of unfavorable FED and RTT could be considered as a high risk factor for prognosis, and decision making in this clinical condition.

\section{Materials and Methods}

\section{Samples}

The study samples consisted of a total of 169 maxillary (103) and mandibular (66) first and second molars extracted because of hopeless prognosis. Those teeth were suffered from a severely advanced periodontitis with Class III molar FI and (over 75\% alveolar bone loss) in 165 individuals with intact roots, prior to, during, or following periodontal therapy. Informed consent was obtained from each patient. The subjects included males and females of 24 to 84 years of age, with a mean age of $47.8 \pm 7.2$ years. The subjects were collected among individuals treated at the Kaohsiung Medical University, Department of Periodontics, for periodontal therapy. The selection criteria of hopeless molars were: 1 ) conditions incapable to be treated by periodontal surgery; 2) presence of a localized Class III molar FI with a vertical probing depth $>10 \mathrm{~mm}$; 3) presence of vertical and horizontal mobility. The molars restored with crowns or bridges or otherwise damaged were excluded to avoid a defective assessment of morphology of the root trunk and root length. The extracted molars were washed in tap water, and the tooth type was determined subsequently. The collected examples were fixed in formalin solution after removing root deposits with ultrasonic scaling and curettage.

Evaluations of clinical parameters, including Periodontal Probing Depth (PPD), Clinical Attachment Level (CAL), gingival index, loe $\mathrm{H}$, et al. [22] plaque index, Silness J, et al. [23] were made at baseline. The PPD and CAL measurements to the nearest $1.0 \mathrm{~mm}$ were determined. Additional measure- ments of maxillary molars at the buccal, mesial and distal furcation sites as well as mandibular molars at the buccal and lingual furcation sites was also carried out to ensure the degree of molar FI and RTT. Clinical probing of molar FI was made using 3 $\mathrm{mm}$ incrementally calibrated Naber's probes (PO2N, Hu-Friedy Co, Chicago, IL).

\section{Measurements and grading system of RTT}

The FED and RTT of the samples were measured under a stereomicroscopy at $6.5 \mathrm{x}$ magnification video camera using a computer system equipped with a Bioscan Optimas Image Analyzer (BOIA) 4 and Digital Electric Caliper Micro-meter (DECM) Hou GL and Tsai CC [4]. To measure the vertical dimension of RTT at the furcation site, only intact molar roots without true root fusion, caries or restoration, were included in our survey. The measurements of PAL for the intra-furcations were made after the extraction. The measurements of molars included the root length of three roots (mesiobuccal, distobuccal, and palatal), and the vertical height of the buccal root trunk (BRT), Mesial Root Trunk (MRT), and Distal Root Trunk (DRT) for maxillary molars, and those of the BRT and LRT for mandibular molars. Then means and standard deviations of these measurements were calculated.

The cases where there was not possible to ascertain the presence and degree of molar FI and root trunk types via clinical probing and radiographic examinations was excluded from the study. The grading system of RTT were classified into types A, B, and C according to the ratio of root trunk height to the root length as described earlier by $[3,19]$.

\section{Measurements and grading system of FED for the extracted molars}

Clinical records including the patient's age, gender, degree of FI, FED of extracted molars (Figures 1a-1c) which were classified into three grades: (1, FED $<0.55 \mathrm{~mm} ; 2,0.55-0.75 \mathrm{~mm}$; and 3, $>0.75 \mathrm{~mm}$ ) based on the width of the working end of ultrasonic tips and periodontal curettes).

Routine periapical radiographic examinations were taken on molars to ensure the degree of FIs and measurements of ABL apical to the base of bone defects before extraction. Radiographic alveolar bone loss of each molar tooth was calculated in percentages and millimeters. Based on classification of RTT and FED, nine-type topography was illustrated

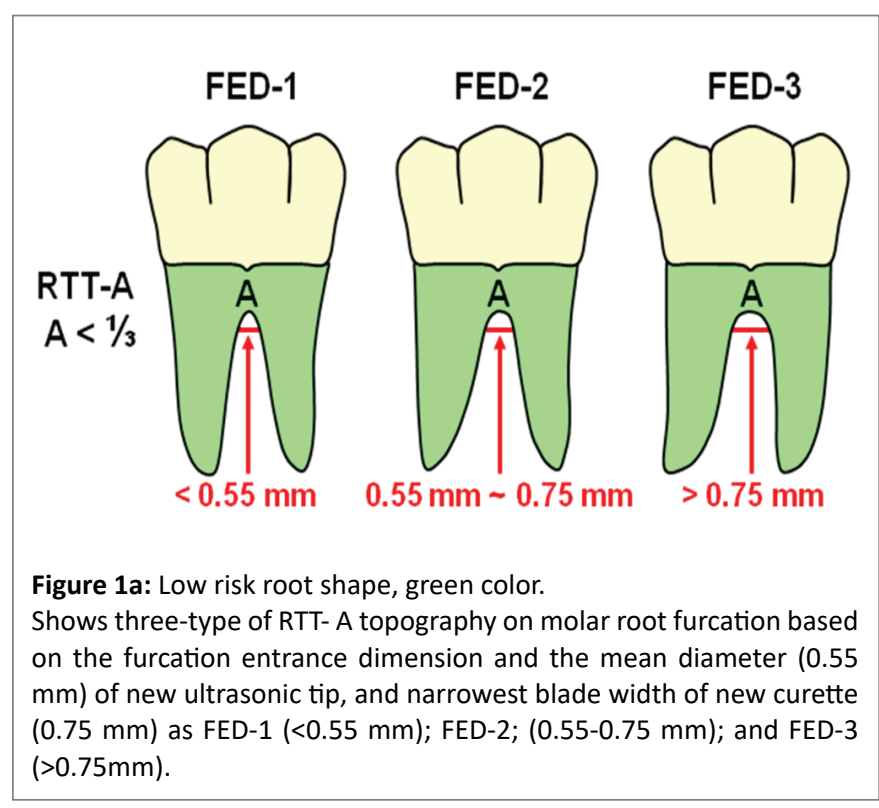




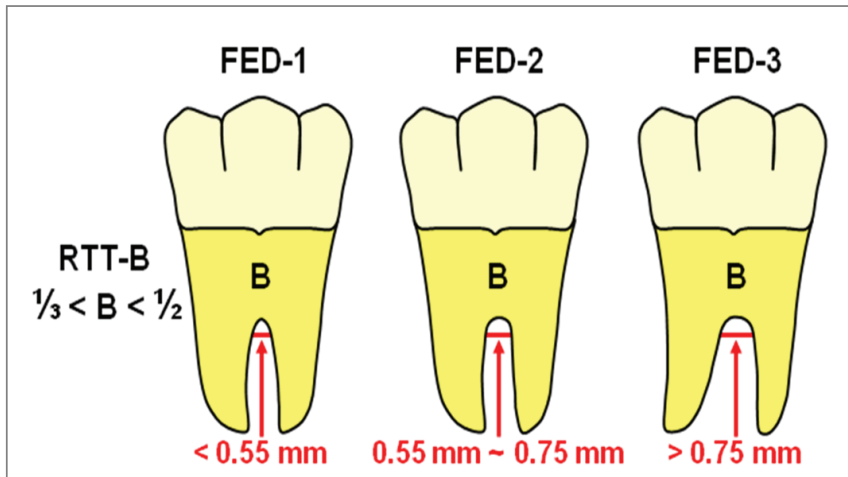

Figure 1b: Median risk root shape of both RTT-C and FED-1; yellow color.

Demonstrated three-type of RTT-B topography on molar root furcation, subclassified as mentioned in Figure $1 \mathrm{a}$.

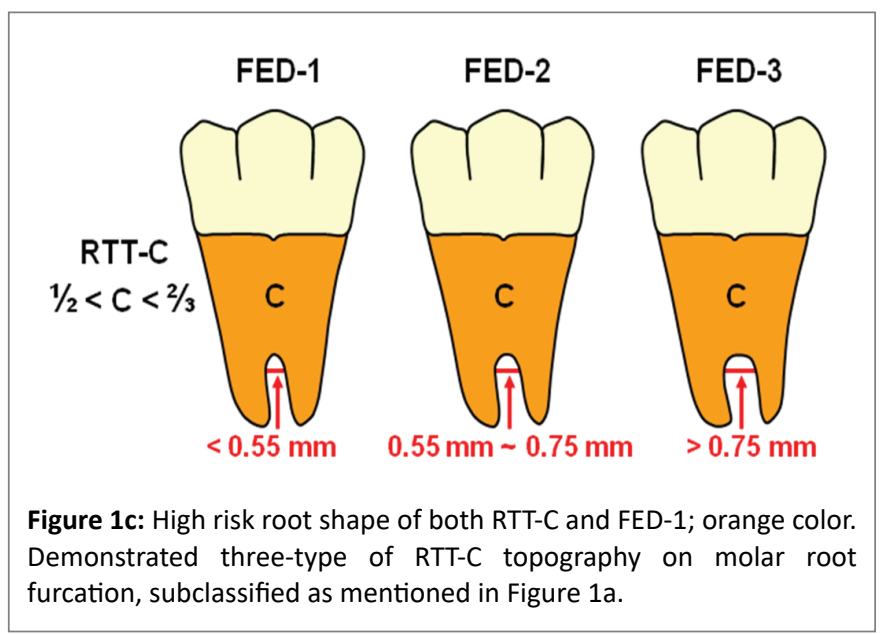

on molar root furcation. The association between each sub-type and the severity of furcation involvement was assessed statistically.

\section{Statistical Analysis}

The means and standard deviations for the root trunk length, root length, and PAL were calculated using SAS/JAMP software. The relationship between the Root Trunk Type (RTT) and FED of molar and at the degree of bone destruction furcation sites among maxillary and mandibular molars were further analyzed using Chi-square tests. Values of $\mathrm{p}<0.05$ were considered as significant level.

\section{Results}

\section{Maxillary molars and FED \& RTT (Table 1)}

First molar: Table 1 presents the distribution and subclasses of FED and RTT for harvested molars. Results showed that the topographic feature of most maxillary first molars were RTT-B \& FED-3 (40.7\%) and RTT-C \& FED-3 (22.2\%) as compared to others. Higher prevalence of extracted teeth was found in the group of RTT-B+FED-3 and RTT$\mathrm{C}+\mathrm{FED}-3$. The difference between the subclasses of FED and RTT at first molar was found to be statistically non-significant $(\mathrm{p}>0.05)$ using the chi-square analysis.

Second molar: Molars with RTT-C \& FED-3 (30.5\%) and RTT-C \& FED-1 (21.8\%) were the most commonly observed as compared to others (Table 1). The prevalence of extracted molars with FED-1 and FED-3 was more commonly associated with RTT-C (21.8\% in FED-1; $30.5 \%$ in FED-3) than with RTT-B (11.5\% in FED-1; $17.2 \%$ in FED-3) and RTT-A (3.5\% in FED-1; 3.5\% in FED-3). The difference between grades of FED and types of RT was statistically non-significant $(\mathrm{p}>0.05)$.

First molar vs. second molar: In addition, extracted teeth with RTT-C+FED-1 tended to occur more commonly at the second molar $(21.8 \%)$ than those at first molar (2.9\%), whereas RTT-C \& FED-3 was both predominant on the second molar $(30.5 \%)$ and first molar (22.2\%). The prevalence of extracted molar roots with RTT-C \& FED$3(30.5 \%)$ and RTT-C \& FED-1 (21.8\%) at the second molar tended to occur more frequently than at the first molar (RTT-C \& FED-3 $=22.2 \%$; RTT-C \& FED-1=2.9\%), but the prevalence of roots with RTT-B \& FED-3 at the second molar (17.2\%) was less than at the first molar (40.7\%). These findings indicated that the hopeless second molars are characterizing as having a rather high prevalence $(21.8 \%)$ of long root trunk length and narrow furcation entrance dimension as compared to the first molar (2.9\%).

\section{Mandibular molars and FED \& RTT (Table 1)}

First molar: Table 1 illustrates the distribution and subclasses of FED and RTT for harvested molars. Current limited data showed that the topographic feature of first molars belonged to RTT-A+FED-3 (50.0\%) and RTT-B+FED-3 (30.8\%). These molars seemed to have a high prevalence of roots with short RTT and wide FED. The difference between the subclasses of FED and RTT at the first molar was found to be statistically non-significant $(\mathrm{p}>0.05)$ using the chi-square analysis.

Second molar: Molars associated with RTT-C+FED-1 and RTTC+FED-2 were $33.0 \%$ and $17.9 \%$, respectively, which were the most commonly observed conditions. The prevalence of extracted molars associated with FED-1 and FED-2 was more commonly found in RTT-C (33.0\% in FED-1; 17.9\% in FED-2) than either RTT-B (15.1 \% in FED-1; $12.3 \%$ in FED-2) or RTT-A (0.9 \% in FED- $1 ; 4.7$ \% in FED2 ). The difference between subclasses of FED and RTT was statistically significant $(\mathrm{p}=0.0042)$. The hopeless molars are characterized as a higher prevalence of roots with long root trunk length and narrow furcation entrance dimension.

First molar $\boldsymbol{v s}$ second molar: In addition, extracted teeth with long root trunk and narrow fraction entrance (RTT-C \& FED-1) tended to occur more commonly at the second molar $(33.0 \%)$ than at the first molar (0\%), whereas RTT-C \& FED-2 tended to occur more frequently at the second molar $(17.9 \%)$ as compared to at the first molar $(0 \%)$.

\section{Analysis among subclasses of FED and RTT in different furcation sites of maxillary molars-Table 2}

First molar: The majority of the extracted maxillary first molars were characterized as having high prevalence of root topography with long root trunk \& wide furcation entrance dimension. This trend was disclosed in groups of RTT-B+FED-3 and RTT-C+FED-3 were found at the mesial (51.1 \% and $28.9 \%$ ) and distal (51.1 \% and $31.1 \%)$ surfaces of the maxillary first molars, respectively (Table 2). The buccal surfaces of furcation sites at the maxillary first molar did not exhibit specific prevalence of root morphology. No statistical difference were found to be ( $p>0.05)$ using the chi-square analysis.

Second molar: On the buccal furcation sites, the majority of extracted molars were closely associated with narrow FED in the moderate and long RTT group (Table 2). This trend was related in the groups of RTT-B+FED-1 (29.3\%) and RTT-C+FED-1 (39.7\%) 
Table 1: Distribution and relationship between Furcation Entrance Dimension and root trunk type in the maxillary and mandibular first and second molars.

\begin{tabular}{|c|c|c|c|c|c|c|}
\hline Molar Location & RTT & $n(\%)$ & $\begin{array}{c}\text { FED-1 } \\
\mathrm{n}(\%)\end{array}$ & $\begin{array}{c}\text { FED-2 } \\
\mathrm{n}(\%)\end{array}$ & $\begin{array}{c}\text { FED-3 } \\
\mathrm{n}(\%)\end{array}$ & Chi-Square Test \\
\hline \multirow{4}{*}{$16 \& 26$} & RTT-A & 24 & $7(5.1)$ & $4(2.9)$ & $13(9.6)$ & $d f=4$ \\
\hline & RTT-B & 74 & $11(8.2)$ & $8(5.9)$ & $55(40.7)$ & $X 2=5.265$ \\
\hline & RTT-C & 37 & $4(2.9)$ & $3(2.2)$ & $30(22.2)$ & $p>0.05$ \\
\hline & Total & 135 & $22(16.3)$ & $15(11.0)$ & $98(72.6)$ & NS \\
\hline \multirow{4}{*}{$17 \& 27$} & RTT-A & 12 & $6(3.5)$ & $0(0)$ & $6(3.5)$ & $d f=4$ \\
\hline & RTT-B & 59 & $20(11.5)$ & $9(5.2)$ & $30(17.2)$ & $X 2=2.669$ \\
\hline & RTT-C & 103 & $38(21.8)$ & $12(6.9)$ & $53(30.5)$ & $p>0.05$ \\
\hline & Total & 174 & $64(36.8)$ & $21(12.1)$ & $89(51.1)$ & NS \\
\hline \multirow{4}{*}{$36 \& 46$} & RTT-A & 15 & $1(3.9)$ & $1(3.9)$ & $13(50.0)$ & $d f=4$ \\
\hline & RTT-B & 9 & $0(0)$ & $1(3.9)$ & $8(30.8)$ & $X 2=0.561$ \\
\hline & RTT-C & 2 & $0(0)$ & $0(0)$ & $2(7.8)$ & $p>0.05$ \\
\hline & Total & 26 & $1(3.9)$ & $2(7.8)$ & $23(88.3)$ & NS \\
\hline \multirow{4}{*}{$37 \& 47$} & RTT-A & 12 & $1(0.9)$ & $5(4.7)$ & $6(5.7)$ & $\mathrm{df}=4$ \\
\hline & RTT-B & 33 & $16(15.1)$ & $13(12.3)$ & $4(3.8)$ & $X 2=15.252$ \\
\hline & RTT-C & 61 & $35(33.0)$ & 19(17.9) & $7(6.6)$ & $p=0.0042^{* *}$ \\
\hline & Total & 106 & $52(49.1)$ & $37(34.9)$ & $17(16.0)$ & $P<0.005$ \\
\hline
\end{tabular}

NS: $p>0.05$

Table 2: Demographic distribution and analysis between FED and RTT in the buccal, mesial and distal surfaces of extracted maxillary first and second molars.

\begin{tabular}{|c|c|c|c|c|c|c|c|}
\hline Molar Location & Root Surface & RTT & $n(\%)$ & $\begin{array}{c}\text { FED-1 } \\
\text { n(\%) }\end{array}$ & $\begin{array}{c}\text { FED-2 } \\
\mathrm{n}(\%)\end{array}$ & $\begin{array}{c}\text { FED-3 } \\
\text { n(\%) }\end{array}$ & Chi-Square Test \\
\hline \multirow{12}{*}{$16 \& 26$} & \multirow{4}{*}{ Buccal } & RTT-A & $13(28.9)$ & $7(15.6)$ & $4(8.9)$ & $2(4.2)$ & $d f=4$ \\
\hline & & RTT-B & $25(55.6)$ & $11(24.4)$ & $5(11.1)$ & $9(20.0)$ & $X 2=0.659$ \\
\hline & & RTT-C & $7(15.5)$ & $3(6.7)$ & $1(2.2)$ & $3(6.7)$ & $p>0.05$ \\
\hline & & Total & $45(100)$ & $21(46.7)$ & $10(22.2)$ & $14(31.1)$ & NS \\
\hline & \multirow{4}{*}{ Mesial } & RTT-A & $5(11.1)$ & $0(0)$ & $0(0)$ & $5(11.1)$ & $d f=4$ \\
\hline & & RTT-B & $25(55.6)$ & $0(0)$ & $2(4.4)$ & $23(51.1)$ & $X 2=0.645$ \\
\hline & & RTT-C & $15(33.3)$ & $0(0)$ & $2(4.4)$ & $13(28.9)$ & $p>0.05$ \\
\hline & & Total & $45(100)$ & $0(0)$ & $4(8.9)$ & $41(91.1)$ & NS \\
\hline & \multirow[t]{4}{*}{ Distal } & RTT-A & $6(13.3)$ & $0(0)$ & $0(0)$ & $6(13.3)$ & $\mathrm{df}=4$ \\
\hline & & RTT-B & $24(53.4)$ & $0(0)$ & $1(2.2)$ & $23(51.1)$ & $X 2=0.575$ \\
\hline & & RTT-C & $15(33.3)$ & $1(2.2)$ & $0(0)$ & $14(31.1)$ & $p>0.05$ \\
\hline & & Total & $45(100)$ & $1(2.2)$ & $1(2.2)$ & $43(95.5)$ & NS \\
\hline \multirow{12}{*}{$17 \& 27$} & \multirow{4}{*}{ Buccal } & RTT-A & $6(10.3)$ & $6(10.3)$ & $0(0)$ & $0(0)$ & $\mathrm{df}=4$ \\
\hline & & RTT-B & $25(43.1)$ & $17(29.3)$ & $3(5.2)$ & $5(8.6)$ & $X 2=0.373$ \\
\hline & & RTT-C & $27(46.6)$ & $23(39.7)$ & $2(3.4)$ & $2(3.4)$ & $p>0.05$ \\
\hline & & Total & $58(100)$ & $46(79.3)$ & $5(8.6)$ & $7(12.0)$ & NS \\
\hline & \multirow{4}{*}{ Mesial } & RTT-A & $3(5.2)$ & $0(0)$ & $0(0)$ & $3(5.2)$ & $\mathrm{df}=4$ \\
\hline & & RTT-B & $13(22.4)$ & $0(0)$ & $2(3.4)$ & $11(19.0)$ & $X 2=0.460$ \\
\hline & & RTT-C & $42(72.4)$ & $7(12.1)$ & $5(8.6)$ & $30(51.7)$ & $p>0.05$ \\
\hline & & Total & $58(100)$ & $7(12.1)$ & $7(12.1)$ & $44(75.9)$ & NS \\
\hline & \multirow{4}{*}{ Distal } & RTT-A & $4(6.9)$ & $0(0)$ & $0(0)$ & $4(6.9)$ & $\mathrm{df}=4$ \\
\hline & & RTT-B & $21(36.2)$ & $3(5.2)$ & $4(6.9)$ & $14(24.1)$ & $X 2=0.430$ \\
\hline & & RTT-C & $33(56.9)$ & $9(15.5)$ & $4(6.9)$ & $20(34.5)$ & $p>0.05$ \\
\hline & & Total & $45(100)$ & $12(20.7)$ & $8(13.8)$ & $38(65.5)$ & NS \\
\hline
\end{tabular}

$\mathrm{n}$ : number of each specific furcation sites examined. NS: no significance; NS: $p>0.05$ 
as compared to other groups. In contrast, on the mesial and distal furcation, most of the root topography were characterized as having a high prevalence of roots with long RTT and wide FED as shown in RTT-C+FED-3 of both furcation sites $(51.7 \%$ at the mesial site; $34.5 \%$ at the distal site). The statistical analysis among the group were found to be statistically non-significant $(p>0.05)$ using the chi-square analysis.

First molar vs Second molar: Further comparison disclosed that the mesial and distal furcation sites of the first and second maxillary molars seemed to share the same root topographies (high prevalence of long RTT and wide FED) at the mesial (51.7\%) and distal (34.5\%) surfaces. However, the buccal root morphologies of the maxillary second molars were slight more prominent in long RTT and narrow FED as compared to those of the first molars $(28.9 \%$ at the mesial and $31.1 \%$ at the distal).

\section{Analysis among subclasses of FED and RTT in different furcation sites of mandibular molars-Table 3}

First molar: With respect to the buccal and lingual furcation areas of first molars, predominant occurrence seemed to be in the subclasses of FED-3, no matter the subclasses of RTT. For instance, the prevalence of RTT-A+FED-3 and RTT-B+FED-3 was $46.1 \%$ and $30.8 \%$ at the buccal furcation sites of the first mandibular molar, respectively. Similar trend occurred at comparable sites of the lingual furcation of the mandibular $(53.8 \%$ vs $30.8 \%)$. In contrast, little prevalence was found in subclasses of FED-1 and FED-2 at both buccal and lingual sites. Most of the extracted first and second molars were characterized as having relatively high prevalence of teeth with wide FED, irrespective of both buccal and lingual furcation. Explanation of these data should be cautions because of limited number of harvested mandibular molars.

Second molar: Different pattern of furcational topographies in the extracted second molars were found as compared to those of the first molars. Extracted molars were favorably affected with narrow furcation entrance and long RTT at both sites of the furcation. This trend was shown in the subclasses of FED-1, the prevalence of RTT-C (41.5\%) was greater than those of RTT-B (13.2\%) and RTT-A (0\%) at the buccal furcation. Similar trend was noted at the lingual furcation. The difference among the subclasses of FED and RTT at the buccal furcation (RTT-C+FED-1) was found to be statistically significant $(\mathrm{p}<0.01)$, while it was not at the lingual furcation.

First molar vs. Second molar: Different pattern of furcation topographies in the extracted second molars were found as compared to those of the first molars. Extracted molars were favorably affected with narrow furcation entrance and long RTT at both sites of the furcation (Table 3). No comparison was made on the prevalence between the first and second mandibular molars because of little number of harvested first molars.

\section{Discussion}

The teeth were harvested from patients affected with periodontitis who required periodontal therapy. Therefore, these data might not reflect the condition of general population. Further epidemiological study through a wide survey from the randomized samples was necessary. Radiographic examination alone is incapable of giving detailed information on the three dimensional morphology of molar roots. Previous studies indicated that radiographic examination, direct assessment using BOIA, [2] digital electric caliper micrometer (DECM) [3] and clinical probing might be the methods of choice to investigate attachment loss of Class III FI.

To the best of our knowledge, little or no clinical reports had been published with respect to simultaneous use occurrence of FED subclasses together with RTT in analysis of prognosis for molars with FIs. The present study showed a high prevalence of FED-1 and RTT-C (narrow FED \& long RTT) at the extracted second molar (21.8\% in maxilla, Table 1; 33.0\% in mandible, Table 2) than at the first molar (2.9\% in maxilla, Table 1; $0 \%$ in mandible, Table 2). These findings appears to be consistent with the notions that a high percentage of narrow furcation could not be thoroughly debrided, even using a combination of ultrasonic tips and periodontal curettes, as reported by

Table 3: Demographic distribution and analysis between FED and RTT in the buccal and lingual surfaces of extracted mandibular first and second molars.

\begin{tabular}{|c|c|c|c|c|c|c|c|}
\hline Molar Location & Root surface & RTT & $n(\%)$ & $\begin{array}{c}\text { FED-1 } \\
\mathrm{n}(\%)\end{array}$ & $\begin{array}{c}\text { FED-2 } \\
\mathrm{n}(\%)\end{array}$ & $\begin{array}{c}\text { FED-3 } \\
\text { n (\%) }\end{array}$ & Chi-Square Test \\
\hline \multirow{8}{*}{$36 \& 46$} & \multirow{4}{*}{ Buccal } & RTT-A & $8(61.5)$ & $1(7.7)$ & $1(7.7)$ & $6(46.1)$ & $d f=4$ \\
\hline & & RTT-B & $4(30.8)$ & $0(0)$ & $0(0)$ & $4(30.8)$ & $X 2=0.831$ \\
\hline & & RTT-C & $1(7.7)$ & $0(0)$ & $0(0)$ & $1(7.7)$ & $p>0.05$ \\
\hline & & Total & $13(100)$ & $1(7.7)$ & $1(7.7)$ & $11(84.6)$ & NS \\
\hline & \multirow{4}{*}{ Lingual } & RTT-A & $7(53.8)$ & $0(0)$ & $0(0)$ & $7(53.8)$ & $d f=4$ \\
\hline & & RTT-B & $5(38.5)$ & $0(0)$ & $1(7.7)$ & $4(30.8)$ & $X 2=0.420$ \\
\hline & & RTT-C & $1(7.7)$ & $0(0)$ & $0(0)$ & $1(7.7)$ & $p>0.05$ \\
\hline & & Total & $13(100)$ & $0(0)$ & $1(7.7)$ & $12(92.3)$ & NS \\
\hline \multirow{8}{*}{$37 \& 47$} & \multirow{4}{*}{ Buccal } & RTT-A & $6(11.3)$ & $0(0)$ & $3(5.7)$ & $3(5.7)$ & $\mathrm{df}=4$ \\
\hline & & RTT-B & $13(27.5)$ & $7(13.2)$ & $6(11.3)$ & $0(0)$ & $X 2=5.265$ \\
\hline & & RTT-C & $34(61.2)$ & $22(41.5)$ & $9(17.0)$ & $3(5.7)$ & $p<0.01$ \\
\hline & & Total & $53(100)$ & $29(54.7)$ & $18(34.0)$ & $6(11.3)$ & $\mathrm{S}^{* *}$ \\
\hline & Lingual & RTT-A & $6(11.3)$ & $1(1.9)$ & $2(3.8)$ & $3(5.7)$ & $\mathrm{df}=4$ \\
\hline & & RTT-B & $20(37.8)$ & $9(17.0)$ & $7(13.2)$ & $4(7.5)$ & $X 2=0.392$ \\
\hline & & RTT-C & $27(50.9)$ & $13(24.5)$ & 10(18.9) & $4(7.5)$ & $p>0.05$ \\
\hline & & Total & $53(100)$ & $23(43.5)$ & $19(35.8)$ & $11(20.8)$ & NS \\
\hline
\end{tabular}

$n$ : number of each furcation sites examined. NS: no significance; NS: $p>0.05$ 
several investigators $[2,10,24]$. In addition, the present data supports the hypothesis that the topography of FED and RTT at the second molars has a higher mortality rate than those at the first molars when teeth are affected by advanced furcation involvement [20,21]. The present observations were also commensurate with that of previous clinical studies, which showed that the second molar had a higher mortality rate, more attachment loss, unfavorable crown-to-root ratio, short root length and a taper-shaped root when compared to first molars $[21,25]$. This information may be of value in evaluating the topographic difference at advanced furcation-involved molars between molar types and arch types.

Our recent study on molars indicated that the mesial FEDs (1.04 $\mathrm{mm}$ and $0.9 \mathrm{~mm})$ were the widest, followed by distal $(0.99 \mathrm{~mm}$ and $0.67 \mathrm{~mm})$ and buccal $(0.74 \mathrm{~mm}$ and $0.63 \mathrm{~mm})$ furcation. Furthermore, an interesting finding was that $63 \%$ and $62 \%$ of all FEDs in both maxillary and mandible second molars, respectively, are narrower than $0.75 \mathrm{~mm}$, which is the narrowest blade width of new curette [2,5]. This result suggests that most of the Chinese teeth are at high risk for continued periodontal breakdown because of the difficulty in gaining access to most furcation with standard scaling (mean of ultrasonic scaling tips $=0.56 \mathrm{~mm}$ ) and root planning instruments (dimension of working end of curettes between $0.75 \mathrm{~mm}$ and $1.1 \mathrm{~mm}$ ).

The present study found that FED-1 occurred more frequently in second molars than in first molars, in particular, at the buccal furcation sites of maxillary molars and mandibular second molars. It also confirmed the previous reports published by investigators $[2,13,26]$. Neither of them actually provided the relationship between FED and RTT and its association with molars of Class III FI.

This study is the first to describe the morphology of FED together with RTT associated with advanced molar FI by arch types. In monitoring, the grades of furcation entrance together with the type of root trunk are closely associated with the variations of molar teeth affected by class III FI. It is obvious that majority of FED-3 and RTT-B are at the mesial and distal furcation sites of the maxillary first molars as well as the majority of FED-3 and RTT-C are at the second molars. At the buccal and lingual furcation sites of the mandibular first molar, FED-3 and RTT-A were the most prevalent, whereas FED-1 and RTT-C (highest risk root shape, Figure 1c) were the most prevalent at the mandibular second molar (Table 3 ). Results suggested that molars extracted with class III FI appear to be with narrower FE and longer $\mathrm{RT}$ in the mandibular second molar than in other molars.

The present study revealed that majority of harvested second mandibular molars was detected in both buccal and lingual furcation having a long RTT and a narrow FED (RTT-C+FED-1, Table 3). This finding disclosed that these molars with this type of root topography might be associated with a risk factor which predisposed molars to severe furcation involvement. Still have some hopeless maxillary molars affected class III FI, these characterizing as having wide FED together with longest RTT located at the mesial and distal furcation sites. This is interesting whether different topographies are associated with the region's inaccessibility to cleansing the associated with the region's the microbial plaque by mechanical plaque control at both mesial and distal furcation sites need to be clarified. Exception to this notion was found in maxillary second molars (buccal furcation).

In this study, another interesting finding was the presence of limited molars, affected by Class IIII FI, with short RTT and wide FED on extracted teeth which required extraction for periodontal therapy, especially in the mandible first molars (13 teeth), as compared to other molar types.
Limitations of the present study were other anatomical factors led to early exposure of periodontitis, for the presence of cervical enamel projection, intermediate bifurcation ridge, root groove, and concavities within furcal areas $[5,12,14,16,17,21]$. The clinical significance of RTT has been considered a key factor in both development and success of furcation therapy [27]. The addition of the dimension of furcation entrance complicates further in both the easiness and success of therapy. The shorter the root trunk, the less attachment has to be lost before the furcation is involved. Once the furcation is exposed, teeth with short root trunks may be more accessible to maintenance procedures and may facilitate some surgical procedures.

Alternatively, teeth with unusually long root trunks or fused roots simultaneous combined with narrow FED may not be appropriate candidates for treatment once the periodontal destruction involving molar furcation, and are regarded as the highest risk root topographic shape (Figure 1c).

In conclusion, clinical roles of FED and RTT seem to be an important factor in both treatment planning and evaluation of prognosis. Closely approximated or fused roots have been known to preclude adequate instrumentation and an obstacle to successful therapy. Teeth with wide FED favors more treatment options and can be easily treated. Therefore, molars extracted because of severe periodontitis and specific root topography which may, firstly, function as a pathway to accumulate microbial plaque and calculus into the furcation entrance and aggravate the progress of disease. Secondly, the simultaneous presence of a narrow FED and long RTT topographies complicates manage-ment of conventional and even regenerative therapy, and also periodontal maintenance [6,28-31].

\section{Ethical Approval}

This article does not contain any studies with human participants or animals performed.

\section{Funding}

This research was supported by a grant from the National Science Council, Taiwan, ROC (NSC-86-2314-B-037-058).

\section{Helsinki Declaration}

All procedures performed in this study is not involving human.

\section{Informed Consent}

For this type is not required.

\section{References}

1. Müller HP, Eger T, Lange DE (1995) Management of furcationinvolved teeth. A retrospective analysis. J Clin Periodontol 22: 911-917.

2. Hou GL, Tsai CC (1994) The morphology of root fusion in Chinese adults (I) Grades, types, location, and distribution. J Clin Periodontol 21: $260-264$.

3. Hou GL, Tsai CC (1997) Types and dimensions of root trunk correlating with diagnosis of molar furcation involvements. J Clin Periodontol 24: 129-135.

4. Carnevale G, Pontorierio R, Hurzeler MB (1995) Management of furcation involvement. Periodontology 2000 9: 69-89.

5. Müller HP, Eger T (1999) Furcation diagnosis. J Clin Periodontol 26: 485-498.

6. Pontoriero R, Lindhe J (1995) Guided tissue regeneration in the treatment of degree II furcations in maxillary molars. J Clin Periodontol 22: 756-763. 
7. Pontoriero R, Lindhe J (1995) Guided tissue regeneration in the treatment of degree III furcations in maxillary molars. J Clin Periodontol 22: 810-812.

8. Fleischer HC, Mellonig JT, Brayer WK, Gray JL, Barnelt JD (1989) Scaling and root planning in multi-rooted teeth. J Periodontol 60: 402-409.

9. Caffesse RG, Sweeney PL, Smith BA (1986) Scaling and root planning with and without periodontal flap surgery. J Clin Periodontol 13: 205-210.

10. Parashis AO, Anagnou-Vareltzids A, Demetriou N (1993) Calculus removal from multi-rooted teeth with and without surgical access. II. Comparison between external and fraction surfaces and effect of furcation entrance width. J Clin Periodontol 20: 294-298.

11. Hou GL, Tsai CC, Weisgold AS (1999) Treatment of molar furcation involvement using root separation and a crown and sleeve-coping telescopic denture. A longitudinal study. J Periodontol 70: 10981199.

12. Lalato DC (1975) Some anatomical factors related to furcation involvements. J Periodontol 46: 608-609.

13. Bower RC (1979) Furcation morphology relative to periodontal treatment. Furcation entrance architecture. J Periodontol 50: 23-27.

14. Bower RC (1979) Furcation morphology relative to periodontal treatment. Furcation root surface anatomy. J Periodontol 50: 366374.

15. Pontoriero R, Lindhe J (1997) Guided tissue regeneration in the treatment of degree-III furcation defects in maxillary molars. J Clin Periodontol 68: 1093-1097.

16. Hou GL, Chen SF, Wu YM, Tsai CC (1994) The topography of the furcation entrance in Chinese molars. Furcation entrance dimensions. J Clin Periodontol 21: 451- 456.

17. Hou GL, Tsai CC (1997) Cervical enamel projection and intermediate bifurcational ridge correlated with molar furcation involvements. J Periodontol 68: 687-693.

18. Hou G L, Cheng YM, Tsai CC, Weisgold AS (1998) A new classification of molar furcation involvement based on the root trunk and horizontal and vertical bone loss. Int J Periodontics Restorative Dent 18: $257-265$
19. Hou G L, Hung CC, Tsai C, Weisgold AS (2005) Topographic study of root trunk type on Chinese molars with Class III furcation involvements: molar type and furcation site. Int J Periodontics Restorative Dent 25: 173-179.

20. Svärdström G, Wennström JL (1988) Furcation topography of the maxillary and mandibular first molars. J Clin Periodontol 15: 271275.

21. Hou GL, Tsai CC, Huang JS (1997) Relationship between molar root fusion and localized periodontitis. J Periodontol 68: 313-319.

22. Loe H, Silness J (1963) Periodontal disease in pregnancy. I. Prevalence and severity. Acta Odontol Scan 21: 533-551.

23. Silness J, Loe H (1964) Periodontal disease in pregnancy. II. Correlation between oral hygiene and periodontal condition. Acta Odontol Scand 22: 121-135.

24. Waerhaug J (1980) The furcation problem: Etiology, pathogenesis, diagnosis, therapy and diagnosis. J Clin Periodontol 7: 73-95.

25. Hou GL, Chen SF, Tsai CC (1996) Furcation entrance dimension, divergent angle and length of CEJ to furcation entrance relate to periodontal therapy. Kaohsiung J Med Sci 12: 707-715.

26. Chiu BM, Zee KY, Corbet EF. Holgren CJ (1991) Periodontal implications of furcation entrance dimensions in Chinese first permanent molars. J Periodontol 62: 308-311.

27. Ammons WF, Harrington GW (2002) Furcation: The problem and its management. In: Carranza's Clinical Periodontology, $9^{\text {th }}$ Edition, Newman MG, Takei HH, Carranza FA (Eds) 825-839.

28. Matia JI, Bissada NF, Maybury JE, Ricchetti P (1986) Efficiency of scaling of the molar furcation area with and without surgical access. Int J Periodontics Restorative Dent 6: 24-35.

29. Karring T, Cortellini P (1999) Regereration therapy: Furcation defects. Periodontol 2000 19: 115-137.

30. Novaes AB Jr, Tamani JP, Oliveira PT, Palioto DB, Almeida AL (2001) Root trunk concavities as a risk factor for regenerative procedures of Class II furcation lesions in dogs. J Periodontol 72: 612- 619.

31. Tonetti MS, Lang HP, Cortellini P, Suvan JE, Adriaens P, et al. (2002) Enamel matrix proteins in regenerative therapy of deep intrabony defects. J Clin Periodontol 29: 317-325. 